\title{
The effects of oscillation period on
}

\section{groundwater wave dispersion in a sandy \\ unconfined aquifer: Sand flume experiments \\ and modelling}

\author{
Seyed Mohammad Hossein Jazayeri Shoushtari ${ }^{\text {a }}$, Nick \\ Cartwright $^{\mathrm{b}}$, Peter Nielsen ${ }^{\mathrm{c}}$, Pierre Perrochet ${ }^{\mathrm{d}}$ \\ a, b Griffith School of Engineering, Gold Coast Campus, Griffith University, \\ Queensland 4222, Australia \\ c School of Civil Engineering, The University of Queensland, 4072, \\ Australia \\ ${ }^{\mathrm{d}}$ Centre d'hydrogéologie, Rue Emile-Argand 11,Case postale 158,2009 \\ Neuchâtel, Switzerland
}

Corresponding author: Seyed Mohamad Hossein Jazayeri

Shoushtari, Griffith School of Engineering, Griffith University, Gold Coast, Queensland, 4222, Australia.

(s.jazayerishoushtari@griffith.edu.au,Tel: +61(0)7 5552 7608) 


\section{Abstract}

This paper presents a new laboratory sand flume dataset on the propagation of groundwater waves in an unconfined sandy aquifer with a vertical boundary subject to simple harmonic forcing with a wide range of oscillation period from $10.7 \mathrm{~s}$ to 909s. The data is unique in that it covers a much wider range of non-dimensional aquifer depths, $n \omega d / K$ (where $n$ is the porosity, $\omega$ is the angular frequency, $d$ is the aquifer depth and $K$ is the hydraulic conductivity) than has been previously investigated. Both the amplitude decay rate and rate of increase in phase lag of the water table waves are observed to monotonically increase with increasing oscillation frequency (increasing $n \omega d / K$ ). This is in contrast to existing theoretical dispersion relations which predict: (1) zero phase lag or standing wave behaviour and (2) an asymptotic decay rate as the frequency increases. Possible influences on the experimental data including sand packing, measurement location, finite amplitude wave effects, unsaturated zone truncation and multiple wave mode effects are unable to explain the discrepancy. The data was also compared against numerical solutions of Richards' equation with and without hysteresis and in both cases, the same qualitative behaviour as the analytic solutions described above is found. The discrepancy between data and predictions remains unexplained and highlights a knowledge gap that requires further 
investigation. These findings relate directly to practical applications in the field of surface-groundwater interactions such as the influence of wave forcing of coastal aquifers on contaminant transport, sediment mobility and salt-water intrusion all of which are influenced by the dispersion of the groundwater wave.

Keywords: Groundwater wave; Hysteresis; Unsaturated flow; Seepage face; Richards’ equation 


\section{Introduction}

Coastal aquifers around the world are exploited by a range of uses including agriculture, potable water supply and waste water disposal and these aquifers are subject to the influence of groundwater waves induced by oceanic oscillations (waves and tides). The propagation of groundwater waves has been shown to have important implications for the mixing of oceanic and sub-surface water masses at the coastal margin (e.g. Li et al., 1999; Robinson et al., 2006; Xin et al., 2010) and also the mobility of sediments on beaches (e.g. Elfrink and Baldock, 2002; Xin et al., 2010; Bakhtyar et al., 2011). In particular, the speed of propagation and decay of the water table wave will dictate the magnitude and variation in hydraulic gradients near the boundary which in turn control flow rates and thus the extent of mixing processes such as salt-water intrusion and contaminant transport.

The dispersion of groundwater waves has received theoretical attention in the literature including the influence of non-hydrostatic pressure and capillarity. The simplest case is that of simple harmonic forcing of an unconfined aquifer across a vertical interface,

$$
h_{o}=d+A \cos (\omega t)
$$

where $h_{o}$ is the driving head [L], $d$ is the mean driving head [L], $A$ is the driving head amplitude $[\mathrm{L}]$ and $\omega=2 \pi / T\left[\mathrm{~T}^{-1}\right]$ is the 
oscillation frequency and $T$ is the oscillation period [T]. Under the assumption of small-amplitude oscillations $(A<<d)$, the form of the water table wave in response to this forcing is (e.g. Steggewentz, 1933; Parlange et al., 1984; Nielsen, 1990; Barry et al., 1996; Li et al., 2000b),

$$
\begin{gathered}
\eta(x, t)=A \operatorname{Re}\left\{e^{-k x} e^{i \omega t}\right\}=A \operatorname{Re}\left\{e^{-\left(k_{r}+i k_{i}\right) x} e^{i \omega t}\right\} \\
=A e^{-k_{r} x} \cos \left(\omega t-k_{i} x\right)
\end{gathered}
$$

where $\eta$ is the water table elevation [L] relative to the mean water level and $k=k_{r}+i k_{i}$ is the water table wave number which describes the dispersive properties of the wave where $k_{r}$ is the decay rate $\left[\mathrm{L}^{-1}\right]$ of the water table wave amplitude and $k_{i}$ is the rate of increase in phase lag $\left[\mathrm{L}^{-1}\right]$ of the water table wave with increasing distance landward and $i=\sqrt{-1}$.

Existing wave numbers derived from experimental (sand flume and Hele-Shaw cell) and field observations have been limited to non-dimensional aquifer depths $n \omega d / K<41$ where $n$ is the specific yield [-] and $K$ is the saturated hydraulic conductivity $\left[\mathrm{LT}^{-1}\right]$. To put these values into context, a groundwater wave induced by a semi-diurnal tide in a $10 \mathrm{~m}$ deep sandy beach aquifer ( $T=12.25$ hours; $n=0.3 ; K=4 \times 10^{-4}$ $\mathrm{m} / \mathrm{s}$ ) corresponds to $n \omega d / K=1.1$ whereas a 10 second wave forcing the same aquifer corresponds to $n \omega d / K=4700$. That is, existing theories on water table wave dispersion are yet to be tested against data on the propagation of high frequency groundwater waves (large $n \omega d / K$ ). This paper addresses this 
gap in knowledge and presents a comprehensive new database of wave numbers derived from controlled sand flume experiments with an experimental parameter range of $4<$ $n \omega d / K<415$.

\section{Existing analytical dispersion relations}

The following sections summarise the theoretical development in the literature that has led to a range of water table wave dispersion relations based on the consideration of different physical influences such as vertical flows (nonhydrostatic pressure) and capillarity. All are based on the assumption of small amplitude waves propagating in a homogeneous, isotropic aquifer.

\subsection{Shallow, capillarity free aquifer}

The simplest theory stems from the assumptions of a shallow (i.e. hydrostatic pressure), capillarity free aquifer which leads to the "Bousinessq" wave number valid for $n \omega d / K$ $<<1$ (e.g. Todd, 1959),

$$
k d=\sqrt{i \frac{n \omega d}{K}}
$$

That is, the shallow aquifer theory predicts the rate of decay to be equal to the rate of increase in phase lag $\left(k_{r}=k_{i}\right)$. This is in clear contrast to available field and laboratory data which indicates that $k_{r} \neq k_{i}$ (e.g. Nielsen, 1990; Aseervatham, 1994; 
Kang, 1995; Raubenheimer et al., 1999; Cartwright et al., 2003; Cartwright, 2004; Cartwright et al., 2004).

\subsection{Shallow aquifer with capillarity effects}

Barry et al. (1996) followed the approach of Parlange and Brutsaert (1987) and applied the non-hysteric Green and Ampt (1911) model of the capillary fringe to correct the shallow aquifer theory for capillarity effects and found,

$$
\begin{aligned}
& k_{r}=\sqrt{\frac{n \omega}{2 d}\left[\frac{1}{\sqrt{K^{2}+\left(\omega n H_{\psi}\right)^{2}}}+\frac{\omega n H_{\psi}}{K^{2}+\left(\omega n H_{\psi}\right)^{2}}\right]} \\
& k_{i}=\sqrt{\frac{n \omega}{2 d}\left[\frac{1}{\sqrt{K^{2}+\left(\omega n H_{\psi}\right)^{2}}}-\frac{\omega n H_{\psi}}{K^{2}+\left(\omega n H_{\psi}\right)^{2}}\right]}
\end{aligned}
$$

where $H_{\psi}$ is the equivalent saturated height of the capillary fringe [L] which is found by integrating the effective saturation from the water table upwards,

$$
H_{\psi}=\int_{h}^{\infty} \frac{\theta-\theta_{r}}{\theta_{s}-\theta_{r}} d z
$$

where $h$ is the water table elevation [L], $z$ is the elevation [L] and $\theta$ is the volumetric water content [-] and the subscripts $S$ and $r$ denote saturated and residual quantities respectively.

In essence, the work of Barry et al. (1996) demonstrates that the presence of moisture above the water table acts to 
reduce the dispersion of the water table wave (slower rates of decay and phase lag increase). In other words, the wave number $k$ is smaller with capillarity effects than without.

\subsection{Non-shallow, capillarity free aquifer}

Building on from the experimental Hele-Shaw cell work of Aseervatham (1994), Nielsen et al. (1997) developed a theory quantifying the influence of vertical flow effects (nonhydrostatic pressure) on periodic groundwater flow. First a $2^{\text {nd }}$ order (in $n \omega d / K$ ) dispersion relation was derived as,

$$
k d=\sqrt{\frac{3}{2}} \sqrt{-1+\sqrt{1+\frac{4}{3} i \frac{n \omega d}{K}}}
$$

which was then extended to infinite order,

$$
k d \tan k d=i \frac{n \omega d}{K}
$$

The implications of the exact solution (equation (8)) is that in the high frequency limit $(n \omega d / K \rightarrow \infty)$ : (1) the theory predicts a zero phase lag $\left(k_{i}\right.$ $=0)$ corresponding to a standing wave scenario and (2) the amplitude decay rate has an asymptotic value of $k_{r}=\pi / 2 d$. The present data however will be shown to contradict this with both $k_{r}$ and $k_{i}$ observed to monotonically increase with increasing $n \omega d / K$. 


\subsection{Non-shallow aquifer with capillarity effects}

The non-shallow aquifer theory of Nielsen et al. (1997) (equation (8)) was extended to include capillarity effects via theoretical and empirical approaches as outlined in the following.

\subsubsection{The non-hysteretic Green and Ampt model}

Li et al. (2000a) adopted the theoretical, non-hysteretic Green and Ampt (1911) model of the capillary fringe and derived the following modified form of equation (8),

$$
k d \tan k d=i \frac{n \omega d}{K+i \omega n H_{\psi}}
$$

As per the capillarity free expression (equation (8)), in the high frequency limit this modified equation also predicts zero-phase lag and asymptotic decay rate.

\subsubsection{The hysteretic dynamic effective porosity model}

Nielsen and Perrochet (2000a; b) conducted sand column experiments which indicated that the Green and Ampt (1911) model was unable to replicate the observed relationship between the total moisture in the column and the water table fluctuations. To account for this Nielsen and Perrochet (2000a; b) introduced the concept of a dynamic effective porosity,

$$
n_{\omega} \frac{\partial h}{\partial t}=n \frac{\partial h_{t o t}}{\partial t}
$$

where $n_{\omega}$ is the dynamic effective porosity [-], $h$ is the water table elevation [L] and $h_{\text {tot }}$ is the equivalent saturated height of 
moisture in the vertical $=d+H_{\psi}[\mathrm{L}] . n_{\omega}$ is complex in nature to account for the fact that fluctuations in $h_{\text {tot }}$ are observed to be both damped $\left(\left|n_{\omega}\right|\right)$ and lag $\left(-\operatorname{Arg}\left\{n_{\omega}\right\}\right)$ those in the water table h (cf. Figure 4, Nielsen and Perrochet, 2000a; b).

Nielsen and Turner (2000) and Cartwright et al. (2005) conducted additional sand column experiments over a wider range of oscillation frequencies and three sediment sizes leading to the following empirical formula for the dynamic effective porosity,

$$
n_{\omega}=\frac{n}{1+2.5\left(i \frac{n \omega H_{\psi}}{K}\right)^{2 / 3}}
$$

The experimental values for $n_{\omega}$ are estimated based on the observed frequency response function of the water table dynamics (cf. equation (21) of Nielsen and Perrochet ,2000a; b) and so any influence of hysteresis on the water table dynamics are implicitly accounted for by equation (11). The dynamic effective porosity concept therefore provides a convenient means of parameterising the influence of hysteresis on the dispersion of groundwater waves by simply replacing the porosity (n) with $n_{\omega}$ into either the shallow or non-shallow dispersion relations (equations (3) and (8) respectively).

As per the capillarity free expression (equation (8)), in the high frequency limit $(n \omega d / K \rightarrow \infty)$ this model also predicts zero-phase lag $\left(k_{i} d=0\right)$ and asymptotic decay rate $\left(k_{r} d \rightarrow \pi / 2\right)$. 


\subsection{Influence of horizontal flow in the unsaturated zone}

As summarised above, the influence of capillarity effects on groundwater wave dispersion has been estimated using either the Green and Ampt (1911) model (equations (4), (5) and (9)) or the empirical dynamic effective porosity (equation (11)) derived from vertical sand column experiments. Both of these approaches only consider vertical flows above the water table. Kong et al. (2013) recently quantified the influence of horizontal flows in the unsaturated zone of the wave number by finding approximate perturbation solutions to the $2 \mathrm{D}$ vertical Richards' equation with Gardner's (1958) moisture-retention and hydraulic conductivity function. They present solutions with first and second order corrections for vertical flow in the unsaturated zone but did not consider vertical flows in the saturated zone (for brevity here, the reader is referred to their equation 20 a through $h$ ),

An alternative approach to account for both the influence of horizontal flow in the unsaturated zone and vertical flow effects in the saturated zone is to follow the approach of Nielsen et al. (1997) but to apply the flow equations up to the equivalent height of total moisture (i.e. to $d+H_{\psi}$ ) which yields the modified dispersion relation,

$$
k\left(d+H_{\psi}\right) \tan \left[k\left(d+H_{\psi}\right)\right]=\operatorname{in} \omega \frac{d+H_{\psi}}{K}
$$


Both the Kong et al. (2013) and equation (12) approaches however predict zero phase lag and asymptotic decay rates for large $n \omega d / K$.

\section{Experimental setup and procedures}

\subsection{The flume}

The new data presented herein was collected from two similarly configured rectangular laboratory sand flumes with unconfined aquifer flow (cf. Figure 1). The original data was collected at The University of Queensland (UQ) in a flume of length $8.9 \mathrm{~m}$ and subsequent reproduction of the experiments was conducted in the flume at Griffith University (GU) with a length of $9.2 \mathrm{~m}$. Both flumes are $1.5 \mathrm{~m}$ high and $0.15 \mathrm{~m}$ wide with no flow boundaries at the landward end and at the bottom which is horizontal. The top of the flume had free connectivity with the atmosphere but was covered in loose plastic to avoid any dust settlement. At the “ocean” end, a driving head reservoir provided simple harmonic forcing of the aquifer across a vertical boundary with an adjustable amplitude and frequency.

\subsection{The sand}

The aquifer sands used in both flumes were well-sorted locally mined quartz sand with the physical and hydraulic characteristics as summarised in Table 1 . The sand was added 
to a water filled flume and manually mixed whilst settling to minimise any air encapsulation or layering effects due to differential settlement. The well-sorted nature of the sand $\left(d_{90} / d_{10} \approx 2\right.$ and $\left.d_{60} / d_{10} \approx 1.5\right)$ and the controlled packing method described above ensured that the aquifer can be considered homogeneous and isotropic.

The properties of the sand used in the UQ flume are those determined by Nielsen and Perrochet (2000a; b). The hydraulic conductivity was estimated based on inverse modelling of the governing equation for their sand column experiments (cf. their equation 13). The equivalent saturated height of the unsaturated zone for the UQ sand was estimated based on their first drying curve data according to,

$$
H_{\psi}=\int_{0}^{\infty} \frac{\theta-\theta_{r}}{\theta_{s}-\theta_{r}} d \psi
$$

where $\psi$ is the suction head [L] which yields $H_{\psi}=0.55 \mathrm{~m}$. In contrast, Cartwright et al. (2009) conducted sand column experiments which measured the magnitude of the drop in pore pressure due to meniscus formation at the sand surface and estimated $H_{\psi} \approx 0.4 \mathrm{~m}$.

For the GU sand, the hydraulic conductivity of the sand was estimated in-situ by measuring the flow rate through the flume driven by a steady hydraulic gradient set by static head levels at either end of the flume and suitably rearranging the Dupuit equation (e.g. Fetter, 1994), 


$$
Q=\frac{1}{2} W K\left(\frac{h_{1}^{2}-h_{2}^{2}}{L}\right)
$$

where $Q$ is the measured flow rate $\left[\mathrm{L}^{3} / \mathrm{T}\right], W$ is the width of the flume [L], $K$ is the hydraulic conductivity $\left[\mathrm{LT}^{-1}\right], h_{1}$ and $h_{2}$ are the static head levels at either end of the flume [L] and $L$ is the flume length [L].

\subsection{Pore pressure measurement}

Under non-hydrostatic pressure conditions, direct measurement of the water table position is not practical and so the pore pressure fluctuations below the water table are used here as a proxy for the water table. As will be shown in section 5.1, a qualitative discrepancy between the theory from section 2 and the experimental data exists and so a range of approaches were employed to observe the pore pressure response to the simple harmonic forcing to ensure that the data collection approach was not influencing the results.

The first and second approaches involved the use of an array of piezometers extending horizontally into the sand through the flume wall at an elevation of $z=0.8 \mathrm{~m}$ (the influence of measurement location on observed wave dispersion characteristics is examined in detail in section 5.3). The first method involved connecting the piezometers to externally mounted manometer tubes which were then videoed and analysed to extract head level time series. The second 
approach involved connecting the piezometers directly to pressure transducers to collect the pressure data.

The last approach used was a pressure probe method deployed through the sand surface (cf. Baldock and Holmes, 1996). In brief, the system consists of externally mounted pressure transducers each connected to narrow diameter probes made of $2 \mathrm{~mm}$ ID stainless steel hypodermic tubing. The pore pressure is transmitted through the probes via $12,0.4 \mathrm{~mm}$ holes laser drilled near the tip (3 per $90^{\circ}$ face). Baldock and Holmes (1996) showed that such a system has a natural frequency response of about $35 \mathrm{~Hz}$, sufficiently greater than the highest frequency measured in the present study (ca. $0.1 \mathrm{~Hz}$ ) and thus avoids any potential issues related to instrument response times.

The data collected by all three approaches were consistent with each other and therefore data collection method has not influenced the forthcoming results.

\subsection{Experimental test regime}

A total of 122 experiments were conducted with driving head parameter ranges of: $T=10.7 \mathrm{~s} \rightarrow 909 \mathrm{~s} ; d=0.81 \mathrm{~m} \rightarrow$ $1.17 \mathrm{~m} ; A=0.09 \mathrm{~m} \rightarrow 0.18 \mathrm{~m}$ which covers a range of nondimensional aquifer depths of $n \omega d / K=4 \rightarrow 415$. For each test, the flume was allowed to reach a steady oscillatory state as 
determined by ensuring that pore pressure wave amplitudes and phases from successive periods remained steady.

\subsection{Determination of the groundwater wave number}

The experimental wave numbers can be derived by assuming that the waves propagate with the general small amplitude wave form given by equation (2). This wave form can be suitably rearranged to perform a linear regression on the data (cf. Nielsen, 1990; Cartwright et al., 2003). That is the amplitude profile is given by,

$$
|\eta(x)|=A e^{-k_{r} x} \Rightarrow k_{r} x=-\ln \left[\left|\frac{\eta(x)}{A}\right|\right]
$$

and the phase lag profile is given by,

$$
\phi(x)-\phi(x=0)=-k_{i} x
$$

Therefore by plotting the relevant quantities as a function of $x$, the slope of the lines of best fit will yield the real and imaginary parts of the wave number. In the case of the experimental data, harmonic analysis is used to determine the amplitudes $(|\eta(x)|)$ and phases $(\phi(x))$.

Figure 2 provides two illustrative examples of the regression analysis for experiments where $T=611 \mathrm{~s}$ and $T=$ 11s. In both instances there is good agreement with equations (15) and (16). For $-\ln [|\eta(x)| / A], R^{2}=0.9981$ and 0.9908 for $T=$ 611s and 11 s respectively and, for $\phi(x)-\phi_{o}, R^{2}=0.9945$ and 0.9394 for $T=611$ s and 11 s respectively. These results confirm 
the suitability of assuming the small amplitude wave form (equation (2)) despite the apparent finite amplitude experimental conditions (average $A / d \approx 0.13$ ).

There is an observed deviation from the linear trend in Figure 2, particularly in the phase lag profiles, where a distinctive downward concave behaviour is observed. This is due to the presence of higher-order wave modes near the boundary which act to increase the overall decay rates and phase lags. These higher wave modes decay much faster than the primary wave mode and so in the interior only the primary mode remains (cf. Nielsen et al., 1997). The influence of higher wave modes on predicted wave numbers is examined further in section 5.6.

\section{Numerical modelling}

The present data was also used to assess the predictive capabilities of a numerical solution of the non-linear Richards' equation,

$$
\frac{\partial \theta}{\partial t}=\frac{\partial}{\partial x}\left[K(\psi) \frac{\partial \Phi}{\partial x}\right]+\frac{\partial}{\partial z}\left[K(\psi) \frac{\partial \Phi}{\partial z}\right]
$$

where $\theta$ is the volumetric soil water content [-], $\Phi=\psi+z$ is the hydraulic head [L], $\psi$ is the pressure head [L], $z$ is the

elevation [L] and $K(\psi)$ is the hydraulic conductivity [LT ${ }^{-1}$ ] (assumed to be uniform and isotropic) which was described using the van Genuchten (1980) function, 


$$
K(\psi)=K_{s} \sqrt{S_{e}}\left\{1-\left[1-S_{e}^{1 / m}\right]\right\}^{2}
$$

where $S_{e}$ is the effective saturation [-] given by,

$$
\begin{array}{ll}
S_{e}=\frac{\theta-\theta_{r}}{\theta_{s}-\theta_{r}} & \text { for } \psi \geq 0 \\
= \begin{cases}1 & \text { for } \psi<0 \\
\frac{1}{\left[1+(\alpha|\psi|)^{\beta}\right]^{m}} & \text { for }\end{cases}
\end{array}
$$

where $\alpha[1 / \mathrm{L}], \beta[-]$ and $m=1-1 / \beta[-]$ are curve fitting parameters.

The Richards' equation was solved using the finite element solver package FEFLOW 6.0 (FEFLOW, 2012). The model domain replicated the sand flume configuration as shown in Figure 1 with no flow boundaries prescribed at the top, bottom and landward end of the flume.

To consider the formation of seepage face at the beach face, a mixed boundary condition was applied using a prescribed head with flux constraint method in the FEFLOW simulations (Shoushtari et al., 2015a). The prescribed head with flux constraint method is similar to Clement et al.'s (1994) method and switches the boundary condition between Dirichlet and Neumann based on the flow direction on each part. This boundary condition is illustrated in Figure 3 where three different zones exist: (1) below the minimum water level the prescribed head is the same as the driving head level $\left(h_{o}\right)$; (2) 
between the minimum and maximum water level, hydraulic head varies with elevation plus a maximum flux constraint; (3) above the maximum water level a no-flow condition is applied.

Initial model results were assessed based on two mesh resolutions: (1) $0.01 \mathrm{~m} \times 0.01 \mathrm{~m}$ and (2) $0.005 \mathrm{~m} \times 0.005 \mathrm{~m}$. The difference between results from the two meshes was less than $2 \%$ confirming that the $0.01 \mathrm{~m} \times 0.01 \mathrm{~m}$ mesh size is fine enough and that the numerical results are independent of the mesh size. The $0.01 \mathrm{~m} \times 0.01 \mathrm{~m}$ discretisation is also consistent with other studies using Richards' equation to model transient unsaturated flow in domains of comparable size (e.g. Clement et al., 1996; Ataie-Ashtiani et al., 1999; Nielsen and Perrochet, 2000a; b; Werner and Lockington, 2003).

A series of simulations were run corresponding to oscillation periods ranging from 10 s to 10000 s $(0.5<n \omega d / K<$ 486). All other forcing parameters remained constant $(A=$ $0.126 \mathrm{~m} ; d=0.887 \mathrm{~m})$ and the adopted hydraulic parameters for the sand corresponded to those presented in row 1 of Table 1 for the UQ sand. To facilitate the comparison with the sand flume data (observations made at $z=0.8 \mathrm{~m}$ ), the wave numbers based on the model results $\Phi(x, z=0.8 \mathrm{~m}, t)$ were obtained using the procedure outlined in section 3.5.

\subsection{Hysteresis modelling}

The effects of hysteresis on periodic water table dynamics has been previously demonstrated for the case of $1 \mathrm{D}$ vertical 
motion (e.g. Lehman et al., 1998; Stauffer and Kinzelbach, 2001; Werner and Lockington, 2003). To examine the influence of hysteresis on groundwater wave dispersion (2D vertical motion), the empirical hysteresis model of Scott et al. (1983) was implemented using FEFLOW (Diersch, 2013) which also solves Richards' equation in conjunction with the van Genuchten (1980) moisture retention formulae as detailed previously in section 4 . For brevity here, the reader is referred to Scott et al. (1983) or Diersch (2013) for further details. It should be noted that using the Scott et. al (1983) hysteresis model causes an artificial pumping error which is produced by the non-closure of hysteresis scanning loops (Kool and Parker, 1987). Parker and Lenhard (1987) eliminated artificial pumping error by enforcing closure of scanning curves but their method is difficult to apply in numerical models since it needs to store many historical reversal points. Werner and Lockington (2006) modified the Parker and Lenhard (1987) method and implemented it in a numerical model and demonstrated showed the significance of the artificial pumping error in terms of moisture-pressure dynamics. In terms of water table wave dynamics however, Shoushtari et al. (2015b) demonstrated that inclusion of hysteresis dynamics in Richards' equation models had no influence on water table wave dispersion for short periods. 
The 2D vertical model domain and boundary conditions were the same as those described in section 4 for the nonhysteretic FEFLOW model. The domain was discretised using a regular grid of rectangular elements $0.01 \mathrm{~m}$ in size. The model employed the same hydraulic conditions as the non-hysteretic FEFLOW model (cf. Table 1). Hysteresis was modelled using a hysteresis ratio of $\zeta=\alpha^{w} / \alpha^{d}=2$ after (Kool and Parker, 1987) where $\alpha^{w}$ and $\alpha^{d}$ correspond to the van Genuchten parameter $\alpha$ (cf. equation (19)) for the wetting and drying curves respectively. The drying curve value $\alpha^{d}$ corresponds to the measured value given in Table 1.

\section{Results and discussion}

\subsection{Wave number theory-data comparison}

Figure 4 provides a summary plot of all experimental wave numbers in comparison with the theoretical dispersion relations outlined in section 2. The data indicates a monotonic increase in wave number with increasing $n \omega d / K$ which is in direct contrast with most of the dispersion relations which predict: (1) zero phase lag $\left(k_{i}=0\right)$ and (2) an asymptotic decay rate corresponding to $k_{r}$ in the range $\pi /\left(2\left[d+H_{\psi}\right]\right)$ and $\pi / 2 d$ for the primary mode.

Cartwright et al. (2003) performed a sensitivity analysis of the dispersion relations outlined in section 2 (cf. their Figure 6) 
and showed that variations in the $n$ and $K$ only act to move the wave number along the existing curve (i.e. the qualitative behaviour of the theories are insensitive to both $n$ and $K$ ). The only parameter which led to a shift in the overall curve position was $H_{\psi}$ but this shift did not alter the qualitative behaviour (i.e. approaching zero phase lag and asymptotic decay rate). Therefore, parameter uncertainty is unable to explain the observed qualitative discrepancy between theory and data.

The observed monotonic increase in wave numbers with increasing $n \omega d / K$ appears to be captured by the $2^{\text {nd }}$ order (in $n \omega d / K)$, capillarity free dispersion relation (equation (7)). However, a more quantitative comparison is shown in Figure 5 where the wave numbers are plotted as a function of $n \omega d / K$ which shows that equation (7) predicts a very rapid initial increase in wave number with increasing $n \omega d / K$ that is not seen in the data.

\subsection{Influence of sand packing}

As described in section 3.1, the well-sorted sand was added to a water filled flume to minimise air encapsulation and layering so it is anticipated that a newly packed flume will be more loosely packed than sand that has been subject to long periods of forcing. The three sets of data shown in Figure 4 are for different packings and whilst some small differences exist, they are all in qualitative agreement with each other (i.e. all 
exhibit monotonically increasing wave numbers with increasing $n \omega d / K)$ and thus flume packing does not explain the qualitative discrepancy between theory and data.

\subsection{Influence of measurement elevation}

Direct measurement of the water table elevation in the flume in the presence of vertical flows (i.e. non-hydrostatic pressure) is not practical and so, as described in section 3.3, the present results have been derived using pore pressure measurements from below the water table as a proxy. The potential influence of this on the experimental wave numbers was examined experimentally by conducting additional experiments where all oscillation parameters were kept constant and only the measurement elevation (relative to the bottom of the flume) was varied. These tests utilised the pressure probe approach as described in section 3.3 and all measurement elevations were in saturated conditions (i.e. $Z_{\text {meas }}$ $\left.<d+H_{\psi}\right)$.

Figure 6 shows the results for three different oscillation periods and in all cases the wave number is seen to increase with measurement elevation. This indicates that pressure wave dispersion is greater higher in the aquifer (near the water table) than lower in the aquifer. Hence, measurement elevation does not contribute to the qualitative discrepancy between theory and data shown in Figure 4 and discussed in section 5.1. 


\subsection{Finite-amplitude effects}

The dispersion relations presented in section 2 were all derived under the assumption of small-amplitude waves where $A<<d$. For the present study the relative amplitude $A / d$ ranged from 0.09 to 0.22 at $x=0 \mathrm{~m}$ which would appear to violate the small amplitude assumption of $A / d<<1$. It is noted that the amplitude decays inside the aquifer (cf. equation (2)) and so the $A / d$ ratio inside the aquifer will exponentially approach smallamplitude behaviour. Also, as shown previously in section 3.5, the experimental wave numbers were extracted using linear regression based on the small-amplitude wave form (equation (2)) which was shown to adequately describe the observed amplitude and phase profiles (cf. Figure 2) and so finite amplitude effects have not contributed to the qualitative discrepancy between theory and data.

\subsection{Influence of unsaturated zone truncation}

In all experiments the sand surface was dry to the touch and remained dry when downward pressure on the surface was applied indicating that the top of the tension saturated zone was also below the sand surface. The mean driving head in the present experiments was around $1 \mathrm{~m}$ and the capillary fringe height $H_{\psi} \approx 0.4 \mathrm{~m}$ which puts the sand surface $\left(z_{s}=1.5 \mathrm{~m}\right)$ just above the tension saturated zone $\left(d+H_{\psi}=1.4 \mathrm{~m}\right)$. However, some truncation of the fully developed unsaturated zone is to be 
expected which will in turn influence moisture dynamics in the unsaturated zone. In the following however it is demonstrated that any truncation effects have not influenced the water table dynamics themselves.

Cartwright et al. (2004) conducted sand column experiments using the same sand and found that truncation effects only started to influence the water table dynamics when,

$$
z_{s} \leq h_{\max }+0.5 H_{\psi}
$$

where $h_{\max }$ is the maximum water table elevation.

For the present sand flume data, the mean driving head and amplitude were around $1 \mathrm{~m}$ and $0.17 \mathrm{~m}$ respectively. In conjunction with $H_{\psi}=0.4 \mathrm{~m}$ this leads to, $h_{\text {max }}+0.5 H_{\psi}=1.17+0.5 \times 0.4=1.37 \mathrm{~m}$ which is less than $z_{s}=$ $1.5 \mathrm{~m}$. This however is a "worst case scenario" in the context of potential truncations effects. Once the pressure wave enters the sand its amplitude decays exponentially (cf. equation (2)) and therefore, inside the sand, $h_{\max }<1.17 \mathrm{~m}$ and $h_{\max }+0.5 H_{\psi}<$ $1.37 \mathrm{~m}$, i.e. even further below the $z_{s}=1.5 \mathrm{~m}$ threshold. Therefore truncation of the unsaturated zone is not considered to be an influencing factor in the present experiments.

The sand column experiments of Cartwright et al. (2004) demonstrated that when truncation effects start to become significant this is manifested in a rapid reduction in the effective porosity. That is, when the capillary fringe is truncated then very little moisture exchange is required to 
facilitate a large change in pore pressure. In terms of water table wave dispersion, a reduced effective porosity leads to smaller wave numbers (slower decay and reduced phase lag) relative to the non-truncated case (larger effective porosity). It follows then that if truncation effects were influencing the present data it would be expected that the measured decay rates and phase lags to be smaller than the analytical solutions (which have no truncation) but the opposite is seen in Figure 4.

In summary, truncation of the unsaturated zone due to proximity of the sand surface may influence unsaturated moisture dynamics but based on the experimental criteria (equation (20)) any truncation effects have not influenced the water table dynamics (and hence water table wave numbers). A more detailed discussion of the influence of truncation effects on moisture dynamics and water table dynamics is provided in the discussion between Hilberts and Troch (2006) and Cartwright et al. (2006).

\subsection{Influence of higher wave modes}

As outlined in section 2.3, Nielsen et al. (1997) found that many wave modes are required in order to match the nonhydrostatic interior with the hydrostatic boundary condition. The exact curves shown in Figure 4 all correspond to the first wave mode. All subsequent wave modes occur to the right of the first mode (cf. Figure 3 of Nielsen et al., 1997) and thus have higher decay rates. The analysis of the data however 
implicitly encompasses all wave modes and so the following analysis examines the influence of higher wave modes on the overall dispersive characteristics of the water table wave.

Considering a non-shallow (non-hydrostatic) aquifer driven by small amplitude, simple harmonic driving head (equation (1)) across a vertical interface, Nielsen et al. (1997) obtained the following solution for the water table,

$$
\eta(x, t)=\sum_{j=1}^{\infty} B_{j} e^{-k_{j} x} e^{i \omega t}
$$

where the coefficient $B_{j}$ is the head coefficient for the $j$ th wave mode given by,

$$
B_{j}=\frac{4 \eta_{0} \sin \left(k_{j} d\right) \cos \left(k_{j} d\right)}{2 k_{j} d+\sin \left(2 k_{j} d\right)}
$$

and $k_{j}$ is the wave number of the $j$ th wave mode determined using the exact small-amplitude non-shallow dispersion relation,

$$
k_{j} d \tan k_{j} d=i \frac{n \omega d}{K}
$$

The corresponding solution for the piezometric head below the water table is,

$$
h^{*}(x, z, t)=\sum_{j=1}^{\infty} B_{j} e^{-k_{j} x} \frac{\cos k_{j} z}{\cos k_{j} d} e^{i \omega t}
$$

The overall "aggregate" wave number influenced by multiple wave modes is then found by applying the approach outlined in section 3.5 to the predicted time series from equation (24). 
Figure 7 shows the resultant curves after including 2000 wave modes (as required to ensure convergence of the summation at $x=0 \mathrm{~m}$ ) with capillarity (via $n=n_{\omega}$, equation (11)) and without $\left(n=n_{e}\right)$. The results show some improvement in the prediction of larger decay rates $\left(k_{r}\right)$ but the theory still approaches zero phase lag $\left(k_{i}=0\right)$ for large $n \omega d / K$ which is not evident in the data. Thus the inclusion of multiple wave modes when predicting the dispersion characteristics does not explain the qualitative discrepancy between theory and data.

\subsection{Richards' equation-data comparison}

Figure 8 compares the numerical results with both the experimental data and theoretical dispersion relations. Whilst the inclusion of hysteresis effects have led to some small quantitative differences when compared to the non-hysteretic results, both sets of results show the same qualitative discrepancy when compared to the data as was seen with the analytic solutions. Interestingly, it is noted that Werner and Lockington (2003) demonstrated the ability of the Richards' equation to accurately simulate $1 \mathrm{D}$ vertical periodic flow sand column experiments after the effects of hysteresis were included. 


\section{Conclusions}

A comprehensive data set on the propagation of groundwater waves in an unconfined laboratory aquifer has been presented which covers a much wider range of nondimensional aquifer depths $(4<n \omega d / K<415)$ than has been previously studied. The groundwater waves were generated by simple harmonic forcing across a vertical boundary and are shown to behave consistently with the form of a small amplitude wave despite $A / d \approx 0.13$. Pore pressure data was analysed to examine the dispersive characteristics of the wave by extracting the complex wave number $k=k_{r}+i k_{i}$ where $k_{r}$ represents the amplitude decay rate and $k_{i}$ represents the rate of increase in phase lag as the wave travels into the aquifer.

The data indicates that there is a monotonic increase in both $k_{r}$ and $k_{i}$ with increasing $n \omega d / K$ which is in contrast with existing theoretical dispersion relations which predict (1) zero phase lag $\left(k_{i} d=0\right)$ and (2) asymptotic decay rate $\left(k_{r} d \rightarrow \pi / 2\right)$ in the limit as $n \omega d / K \rightarrow \infty$.

The influence of sand packing, measurement location, finite wave amplitude effects, unsaturated zone truncation and higher wave modes have all been eliminated as possible causes of the discrepancy. Numerical solution of the 2D vertical Richards' equation also exhibited the same qualitative discrepancy and thus any non-linear effects cannot explain the observed discrepancy. 
The new experimental data has identified a qualitative discrepancy between the experimental data and analytic solutions and numerical solution of Richards' equation (with and without hysteresis effects) for large values of $n \omega d / K$. The cause of the discrepancy remains unexplained which has implications for the practical application of these models in surface-groundwater flow problems such as the influence of wave forcing of coastal/estuarine aquifers on subterranean mixing and sediment mobility. Examination of these types of problems all rely on accurate prediction of the driving pressure gradients which are ultimately controlled by the dispersion of the groundwater wave.

\section{Acknowledgements}

The first author has been supported by Griffith University International Postgraduate Research Scholarship and a Griffith University Postgraduate Research Scholarship. Data in this paper can be obtained by contacting the corresponding author.

\section{References}

Aseervatham, A. M. (1994), Tidal Dynamics of Coastal Watertables, Ph.D. Thesis thesis, 253 pp, University of Queensland, Brisbane, Australia. 
Ataie-Ashtiani, B., R. E. Volker, and D. A. Lockington (1999), Numerical and experimental study of seepage in unconfined aquifers with a periodic boundary condition, Journal of Hydrology, 222, 165-184.

Bakhtyar, R., A. Brovelli, D. A. Barry, and L. Li (2011), Waveinduced water table fluctuations, sediment transport and beach profile change: Modeling and comparison with large-scale laboratory experiments, Coastal Engineering, 58(1), 103-118, doi: http://dx.doi.org/10.1016/j.coastaleng.2010.08.004.

Baldock, T. E., and P. Holmes (1996), Pressure gradients within sediment beds, paper presented at Proc. 25th Intl. Conf. on Coastal Engineering, ASCE, Orlando, Florida.

Barry, D. A., S. J. Barry, and J.-Y. Parlange (1996), Capillarity correction to Periodic solutions of the shallow flow approximation, in Mixing in Estuaries and Coastal Seas, Coastal and Estuarine Studies, edited by C. B. Pattiaratchi, pp. 496-510, AGU, Washington DC.

Cartwright, N. (2004), Groundwater dynamics and the salinity structure in sandy beaches, $\mathrm{PhD}$ Thesis thesis, $211 \mathrm{pp}$, University of Queensland, Brisbane. 
Cartwright, N., P. Nielsen, and S. L. Dunn (2003), Watertable waves in an unconfined aquifer: Experiments and modelling, Water Resources Research, 39(12), 1330.

Cartwright, N., P. Nielsen, and L. Li (2004), Experimental observations of watertable waves in an unconfined aquifer with a sloping boundary, Advances in Water Resources, 27, 9911004.

Cartwright, N., P. Nielsen, and P. Perrochet (2005), The influence of capillarity on a simple harmonic oscillating water table: sand column experiments and modelling, Water Resources Research, 41(8), W08416.

Cartwright, N., P. Nielsen, and P. Perrochet (2006), Reply to comment by A. G. J. Hilberts and P. A. Troch on "Influence of capillarity on a simple harmonic oscillating water table: Sand column experiments and modeling" Water Resources Research, 42(11), W11602, doi: 10.1029/2006WR005188.

Cartwright, N., P. Nielsen, and P. Perrochet (2009), Behaviour of a shallow watertable under periodic flow conditions, Water Resources Research, 45(W03416), doi: 10.1029/2008WR007306. 
Clement, T. P., W. R. Wise, F. J. Molz, and M. Wen (1996), A comparison of modeling approaches for steady-state unconfined flow, Journal of Hydrology, 161, 189-209.

Diersch, H.-J. G. (2013), FEFLOW: Finite Element Subsurface Flow and Transport Simulation System Reference Manual, 292 pp., DHI-WASY GmbH.

Elfrink, B., and T. E. Baldock (2002), Hydrodynamics and sediment transport in the swash zone: a review and perspectives., Coastal Engineering, 45(3), 149-167.

Fetter, C. W. (1994), Applied Hydrogeology, 3rd ed., 691 pp., Prentice Hall, New Jersey.

Gardner, W. R. (1958), Some steady-state solutions of the unsaturated moisture flow equations with application to evaporation from a water table. , Soil Science, 85(4), 228-232.

Green, W. H., and G. A. Ampt (1911), Studies on Soil Physics 1. The flow of air and water through soils, Journal of Agricaltural Science, IV(I), 1-24.

Hilberts, A. G. J., and P. A. Troch (2006), Comment on “'Influence of capillarity on a simple harmonic oscillating water 
table: Sand column experiments and modeling', by Nick Cartwright et al., Water Resources Research, 42(11), W11601, doi: 10.1029/2006WR005042.

Shoushtari, S. M. H., P. Nielsen, N. Cartwright, and P. Perrochet (2015a), Periodic seepage face formation and water pressure distribution along a vertical boundary of an aquifer, Journal of Hydrology, 523(0), 24-33, doi: http://dx.doi.org/10.1016/j.jhydrol.2015.01.027.

Shoushtari, S. M. H., N. Cartwright, P. Perrochet and P. Nielsen, (2015b), Influence of hysteresis on groundwater wave dynamics in an unconfined aquifer with a sloping boundary Journal of Hydrology, 531(3), 1114-1121, doi: http://dx.doi.org/10.1016/j.jhydrol.2015.11.020.

Kang, H.-Y. (1995), Watertable Dynamics Forced by Waves, PhD Thesis thesis, 200 pp, University of Queensland, Brisbane. Kong, J., C.-J. Shen, P. Xin, Z. Song, L. Li, D. A. Barry, D. S. Jeng, F. Stagnitti, D. A. Lockington, and J.-Y. Parlange (2013), Capillary effect on watertable fluctuations in unconfined aquifers, Water Resources Research, 49(5), 3064-3069, doi: 10.1002/wrcr.20237. 
Kool, J. B., and J. C. Parker (1987), Development and evaluation of closed-form expressionbs for hysteretic soil hydraulic properties, Water Resources Research, 23(1), 105114.

Lehman, P., F. Stauffer, C. Hinz, O. Dury, and H. Flühler (1998), Effect of hysteresis on water flow in a sand column with a fluctuating capillary fringe, Journal of Contaminant Hydrology, 33(1-2), 81-100.

Li, L., D. A. Barry, F. Stagnitti, and J.-Y. Parlange (1999), Submarine groundwater discharge and associated chemical input to a coastal sea, Water Resources Research, 35, 32533259.

Li, L., D. A. Barry, F. Stagnitti, and J.-Y. Parlange (2000a), Groundwater waves in a coastal aquifer: A new governing equation including vertical effects and capillarity, Water Resources Research, 36(2), 411-420.

Li, L., D. A. Barry, F. Stagnitti, J.-Y. Parlange, and D.-S. Jeng (2000b), Beach water table fluctuations due to spring-neap tides: moving boundary effects, Advances in Water Resources, 23, 817-824. 
Nielsen, P. (1990), Tidal dynamics of the water table in beaches, Water Resources Research, 26(9), 2127-2134.

Nielsen, P., and I. Turner (2000), Groundwater waves and water exchange in beaches, paper presented at Proc. 27th Intl. Conf. on Coastal Engineering, ASCE, Sydney, Australia.

Nielsen, P., and P. Perrochet (2000a), Watertable dynamics under capillary fringes: experiments and modelling, Advances in Water Resources, 23(5), 503-515.

Nielsen, P., and P. Perrochet (2000b), ERRATA: Watertable dynamics under capillary fringes: experiments and modelling [Advances in Water Resources 23 (2000) 503-515], Advances in Water Resources, 23(8), 907-908.

Nielsen, P., A. M. Aseervatham, J. D. Fenton, and P. Perrochet (1997), Groundwater waves in aquifers of intermediate depths, Advances in Water Resources, 20(1), 37-43.

Parker, J.C. and Lenhard, R.J. (1987), A model for hysteretic constitutive relations governing multiphase flow. 1. Saturationpressure relations. Water Resources Research, 23, 2187-2196. 
Parlange, J.-Y., and W. Brutsaert (1987), A capillarity correction for free surface flow of groundwater, Water Resources Research, 23(5), 805-808.

Parlange, J.-Y., F. Stagnitti, J. L. Starr, and R. D. Braddock (1984), Free-surface flow in porous media and periodic solution of the shallow-flow approximation, Journal of Hydrology, 70, 251-263.

Raubenheimer, B., R. T. Guza, and S. Elgar (1999), Tidal water table fluctuations in a sandy ocean beach, Water Resources Research, 35(8), 2313-2320.

Robinson, C., B. Gibbes, and L. Li (2006), Driving mechanisms for groundwater flow and salt transport in a subterranean estuary, Geophys Res Lett, 33(L03402), doi:10.1029/2005GL025247.

Scott, P. S., G. J. Farquhar, and N. Kouwen (1983), Hysteresis effects on net infiltration, Advances in Infiltration, Am. Soc. Agri. Eng., 11-83, 163-170.

Stauffer, F., and W. Kinzelbach (2001), Cyclic hysteretic flow in porous medium column: model, experiment, and simulations, Journal of Hydrology, 240(3-4), 264-275. 
Steggewentz, J. H. (1933), The influence of the tidal motion of seas and tidal rivers on the height of rise of the groundwater Delft University of Technology, Delft.

Todd, D. K. (1959), Ground Water Hydrology, 2nd ed., 336 pp., John Wiley, Hoboken, N. J.

van Genuchten, M. T. (1980), A closed form equation for predicting the hydraulic conductivity of unsaturated soils, Soil Science Society of America Journal, 44, 892-898.

Werner, A. D., and D. A. Lockington (2003), Influence of hysteresis on tidal capillary fringe dynamics in a well-sorted sand, Advances in Water Resources, 26(11), 1199-1204.

Werner, A. D., and D. A. Lockington (2006), Artificial pumping errors in the Kool-Parker scaling model of soil moisture hystersis, Journal of Hydrology, 325, 118-133.

Xin, P., C. Robinson, L. Li, D. A. Barry, and R. Bakhtyar (2010), Effects of wave forcing on a subterranean estuary, Water Resources Research, 46(W12505), doi: 10.1029/2010WR009632. 